\title{
Estrategias institucionales de "vinculación universitaria" orientadas al desarrollo local. Reflexiones a partir de un estudio de caso
}

\author{
Estratégias institucionais da universidade para o desenvolvimento local. Reflexões de \\ um estudo de caso
}
Institutional strategies the universities oriented to local development. Reflections from a case study

Stratégies institutionnelles universitaires en faveur du développement local. Réflexions sur une étude de cas

\author{
M. Fernanda Di Meglio* \\ (di_megliofernanda@hotmail.com)
}

\author{
Andres Harispe ${ }^{* *}$ \\ (andresharispe@gmail.com)
}

Recebido em14/05/2014; revisado e aprovado em 10/08/2014; aceito em 24/09/2014

DOI: http://dx.doi.org/10.1590/1518-70122015117

\begin{abstract}
Resumen: El actual modelo de relación entre la universidad argentina y la sociedad local está en proceso de transformación, pasando de un esquema ofertista a una dinámica de involucramiento más activo en las problemáticas locales. En este marco, se desarrolla un estudio de caso, identificando, describiendo y analizando las principales estrategias de vinculación universitaria de una universidad de gestión pública, en pos de una mayor comprensión y reflexión de su función en los procesos de desarrollo local.

Palabras-claves: Universidad. Vinculación. Desarrollo local.

Resumo: O atual modelo de relação entre a universidade ea sociedade local está em transição da um regime "do lado da oferta" a um envolvimento mais "ativo" nos processos de articulação local. Neste contexto, o documento identifica, descreve e analisa as principais estratégias de interação de uma universidade pública em busca de uma maior compreensão do papel da estrtagias para a geração e contribuição em processo de desenvolvimento local. Palavras-chave: Universidade. Vinculação. Desenvolvimento local.

Abstract: The current model of relation between the university and the local community is going through a process of transformation from a "offer-centered" schema to a more "active" involvement dynamic of the local instances of articulation. This paper identifies, describes and analyzes the main strategies of "relation" of one university in order to understand the role of strategies in the generation and contribution to local development.

Key words: University. Relations. Local development

Résumè: Le modèle actuel de la relation entre l'université et la communauté locale est dans le processus de transformation d'un régime "offre" à une participation plus "actif" dans les processus de de développement local. Dans ce contexte, nous développons une étude de cas afin d'identifier, de décrire et d'analyser les principales stratégies de "interaction" de l'université à une meilleure compréhension du rôle des stratégies pour la génération et la contribution au processus de développement local.
\end{abstract}

Mots-clés: Université. Interaction. Développement local.

\section{Introducción}

Actualmente, el vínculo de las universidades con sus entornos socioeconómicos locales y regionales se ha colocado en el foco de la discusión de las políticas de educación superior en Argentina. En este campo, el conjunto de iniciativas puestas en marcha por la Secretaria de Políticas Universitarias (SPU) a partir del año 2003 puso de manifiesto una clara orientación de la política universitaria, reforzando una visión integral acerca de la misión central de la universidad y la necesidad de fortalecer los procesos de construcción y aplicación crítica del conocimiento. Desde esta posición, la universidad intenta reafirmar su autonomía valorizando su rol activo en los procesos de desarrollo (SAGASTIZÁBAL, 2002).

En este marco contextual, con diferentes estilos y capacidades, las universidades públicas argentinas delinearon un conjunto de estrategias de promoción de la vinculación a efectos de transferir y promocionar los

\footnotetext{
* Universidad Nacional del Centro de la Provincia de Buenos Aires, Argentina.

** Universidad Nacional de San Martin (UNSAM). Buenos Aires, Argentina.
} 
avances científicos y tecnológicos en las actividades socio-productivas y promover el interés y la participación del personal académico en dichas actividades. En este sentido, resulta importante no sólo el esfuerzo que hacen las instituciones para generar estructuras de vinculación sino, principalmente, el compromiso que la institución establece para el desarrollo de ésta actividad.

A los efectos de profundizar en el proceso de vinculación universitaria se desarrolla un estudio de caso, la Universidad Nacional del Centro de la Provincia de Buenos Aires (UNCPBA), Argentina, identificando, describiendo y analizando las principales estrategias de vinculación universitaria, en pos de una mayor comprensión y reflexión del rol de las mismas para la generación y contribución a los procesos de desarrollo local. Se parte de considerar que este tipo de estrategias fortalece las relaciones en dos planos: al interno de la universidad y en su relación con la comunidad.

En los estudios sobre la función de vinculación universitaria predominan experiencias vinculadas a las universidades de tamaño grande ${ }^{1}$, por lo cual, la presente propuesta se centra en otro tipo de universidad: una universidad de gestión pública de tamaño mediano ${ }^{2}$ del interior de la Provincia de Buenos Aires, Argentina.

\section{Universidad y desarrollo local}

La mayoría de los estudios sobre el desarrollo local le asignan suma importancia al conocimiento $\mathrm{y}$, por consiguiente, a las instituciones involucradas en su producción y transferencia, como las universidades, institutos de investigación y el sistema científico tecnológico en su conjunto (ROFMAN; VILLAR, 2007). Dentro de este postulado general, se pueden identificar dos vertientes que ponen el acento en distintas dimensiones de la contribución que pueden ofrecer las instituciones del conocimiento a los procesos de desarrollo local.

\footnotetext{
${ }^{1}$ D’Onofrio G. (1998), Naidorf, J y Armella J. (2003); González Carella y Inés Zanfrillo (2004); María Fernanda Juarros (2005); Naidorf, J. (2009).

${ }^{2}$ La clasificación de las universidades por tamaño está basada en una clasificación propuesta por la Red de Vinculación Tecnológica (RedVitec).
}

Para Rofman y Villar (2007) la perspectiva que está más presente en las elaboraciones latinoamericanas propone jerarquizar el papel del conocimiento en los procesos políticos de construcción del proyecto de desarrollo territorial. Se habla aquí de fortalecimiento de la identidad local, ampliación de la esfera pública y democratización de la cultura política, como procesos de orden sociopolítico que requieren de un mayor conocimiento de los actores locales acerca de las condiciones y problemáticas del territorio. En estos enfoques se hace hincapié en "el papel que juegan las instituciones de formación e investigación en la implementación de estrategias participativas de planificación, en tanto mecanismos de decisión sobre las vías de desarrollo de una comunidad, basados en la participación de los actores locales y la socialización del conocimiento" (ROFMAN; VILLAR, 2007, p. 48).

Esta perspectiva pone de manifiesto que "las instituciones dedicadas a la producción y transferencia del conocimiento forman parte del proceso social de construcción del territorio, y que, por lo tanto, no pueden permanecer ajenas a la responsabilidad de participar en la formulación del proyecto político de transformación local o regional" (ROFMAN, 2005, p. 49). Estos atributos le permite a la universidad asumir un lugar de liderazgo o de mediación en los procesos de vinculación multiactoral. De esta forma, el modelo de relación entre la universidad y la sociedad local se encuentra en proceso de transformación, pasando de un esquema de promoción de la oferta tecnológica a una participación activa en las problemáticas del desarrollo.

En este esquema de relacionamiento dos áreas de intervención cobran relevancia dentro de la dinámica local. Por un lado, la labor específica relativa a la oferta de carreras de grado y posgrado así como la investigación referida a procesos de desarrollo local. Por otro lado, se visualiza un modelo de vinculación entre las universidades y el entorno territorial que se diferencia mucho del modelo academicista, puesto que implica la participación en las instancias de vinculación en tanto actor del territorio con intereses propios y modalidades específicas de intervención (ROFMAN, 2005).

A su vez, la localización de algunas universidades, dejando por fuera de este 
análisis a las ubicadas en las metrópolis, permite avanzar en la idea de "universidad del territorio" (HERNÁNDEZ PÉREZ, 2008) entendiendo por este concepto a las instituciones ubicadas en aglomeraciones urbanas de tamaño intermedio (ATI) (VAPÑARSKI; GOROJSVSKY, 1990; VELÁZQUEZ, 2001). El nivel de influencia de estas universidades es significativamente mayor ya que, entre otras razones, aglutina una parte significativa de las personas más calificadas y/o con conocimientos específicos de la región donde está inserta y conoce o tiene mayor contacto con los problemas del territorio donde está localizada que la convierte en una institución clave a nivel local.

En ese sentido, Boisier (2005) sostiene que el papel de las universidades subnacionales resulta de la mayor importancia, no sólo en términos de transferencia tecnológica a procesos fabriles y organizacionales, sino también en su contribución a la creación y difusión de marcos cognitivos nuevos, contemporáneos y pertinentes para dar respaldo científico a las intervenciones de la propia sociedad sobre los dos procesos de cambio social más importantes para ella misma: el crecimiento y el desarrollo territorial.

\subsection{La transformación de las funciones y tipos de universidad}

En este marco, Fernández de Lucio et al. (2000) presenta una clasificación en la cual distingue entre universidad empresarial y emprendedora. Según estos autores, la primera es aquella que considera que los conocimientos tienen un valor de mercado y por lo tanto son susceptibles de ser vendidos, mientras que la segunda, utiliza el conocimiento como un potencial al servicio de su entorno más que como un bien económico objeto de intercambio.

De esta forma, la universidad asume un papel mucho más activo para la discusión y solución de los problemas de la sociedad en la cual se inserta, configurando otro tipo de institución denominada por Fernández de Lucio et al. (2000) como "universidad social".

En consecuencia, necesita disponer de una misión y estrategia de actuación determinada para actuar en dicho contexto de acuerdo con tres objetivos básicos:
- Atender mediante respuestas innovadoras las nuevas demandas de formación,

- Incrementar la actividad de I+D en interacción con el entorno socioeconómico;

- Participar activamente en el desarrollo de la sociedad.

Asimismo, uno de los aspectos centrales de esta nueva misión es la orientación de la investigación hacia prioridades o hacia la solución de problemas económicos y sociales específicos (CASAS, 2001).

\subsection{La función de "vinculación universitaria"}

La función de vinculación surge como una definición propia que pretende diferenciarse del clásico concepto de extensión universitaria. La existencia de vínculos universidad-sociedad no es nueva, sin embargo, ha adquirido un renovado impulso y rasgos particulares. En este sentido, las universidades latinoamericanas, a diferencia de sus pares de otras partes del mundo, constituyen un ejemplo temprano de un modelo de tres roles a partir del movimiento de la Reforma Universitaria de principios de siglo, en el que la tercera responsabilidad estuvo centrada en su relación directa con la sociedad.

En el mismo sentido, Arocena y Sutz (2001) manifiestan que "en América Latina desde hace muchas décadas, la universidad ha planteado que su razón de ser no se atiende cabalmente a menos que junto a la investigación y a la docencia se propenda a cumplir un papel más directo en el ejercicio de su responsabilidad social en tanto productora de conocimientos. En América Latina en todo caso este tercer rol sería el cuarto, siendo el tercero el representado clásicamente por la función de extensión universitaria" (AROCENA; SUTZ, 2001, p. 54).

Es así, que para algunos analistas especializados, esta nueva función es una revisión y redefinición de la tercera función de las universidades latinoamericanas (SUTZ, 1997; DAGNINO; VELHO, 1998) en el sentido de una ampliación tanto cuantitativa como cualitativa de la tradicional extensión social. Desde esta perspectiva, la presente investigación retomará la propuesta de autores como Gibb (1993), Stiles (2002) y Molas Gallart (2005) que definen a esta función como el papel de las instituciones universitarias en relación a 
las necesidades económicas y sociales de su territorio o entorno de referencia con una actuación tanto en dimensiones locales como regionales. Como plantea Campos Ríos y Sánchez Daza (2005) esta nueva propuesta incluye también otros aspectos fundamentales como la noción de que la vinculación no sólo debe darse al exterior sino, sobre todo, al interior de la propia universidad.

\section{Tipología de las estrategias de vinculación universitaria}

En este marco, las universidades públicas argentinas han delineado diferentes estrategias de promoción de la vinculación a efectos de transferir y promocionar los avances científicos y tecnológicos en las actividades socio-productivas y promover el interés y la participación del personal académico en dichas actividades. Existen diferentes maneras de nombrar las iniciativas tomadas para vincular una institución con el entorno territorial. Se les puede llamar actividades, elementos, componentes, procedimientos o estrategias. Utilizamos el término estrategia, pues en ella está inherente la noción de acción dirigida y planeada, además de tener la ventaja de aplicarse tanto a actividades de naturaleza académica como administrativa (JOCELYNE GACEL ÁVILA, 1999).

Las experiencias recientes suelen combinar elementos propios de distintas estrategias y, más aún, cuando confluyen estrategias institucionales diferentes y/o complementarias los efectos sinérgicos son más notables. Además es necesario tener en cuenta que estamos frente a procesos que se desarrollan y maduran a lo largo del tiempo, lo que hace también que en su avance se modifiquen, amplíen y enriquezcan las modalidades operativas. Por ejemplo, el punto de partida puede ser la oferta de grupos de investigación, el siguiente paso puede ser la institucionalización de programas integrales con múltiples actores, que, a su vez, puede madurar hacia proyectos asociativos más complejos y de mayor alcance (CIAI et al., 1997).

Si tomamos como referencia la clasificación propuesta por Ciai et al. (1997) los principales tipos de estrategias de promoción de la vinculación en el ámbito local que utilizan las universidades son:

\section{a) Estrategias universitarias orientadas a promover la oferta tecnológica}

Este tipo de estrategia, generalmente, se relaciona a las actividades que las universidades realizan con actores públicos y privados, entre ellas, actividades de capacitación, asistencia técnica, consultoría y transferencia de tecnología. Por ejemplo, los proyectos de capacitación dirigidos a empresarios son uno de los temas más frecuentes de cooperación entre la universidad y el sector productivo. Los proyectos de capacitación suelen ser además la puerta de entrada para el establecimiento de las primeras relaciones entre la universidad y actores externos que luego evolucionan hacia proyectos de cooperación de mayor envergadura (CIAI et al., 1997). Estas estrategias generalmente son actividades puntuales que las universidades realizan en función de su oferta científica tecnológica. Dicha oferta es muy variada y está sujeta a las capacidades específicas de los grupos de investigación y de la propia universidad. En estos casos, las universidades han hecho esfuerzos bastante sistemáticos en la formulación de la oferta de productos y de capacidades disponibles (CIAI et al., 1997).

\section{b) Estrategias universitarias orientadas al promover el desarrollo regional}

En estos casos el objetivo de la estrategia tiene alcance regional y el elemento innovativo más importante es la promoción de la cooperación horizontal entre múltiples actores. Se trata de una modalidad difícil de implementar ya que es necesario superar pautas competitivas y obstáculos institucionales y políticos. Las universidades, sobre todo las implantadas en el interior del país, pueden aportar no sólo las capacidades profesionales y tecnológicas de sus docentes e investigadores sino también contribuir a consolidar prácticas asociativas, jugando un importante rol en el desarrollo regional (CIAI et al., 1997). Asimismo, este tipo de estrategia incentiva la articulación de la oferta y demanda de conocimiento a partir de la cooperación horizontal entre múltiples actores.

\subsection{Los proyectos: tipos y temas}

A su vez, las estrategias que se orientan al desarrollo local se subdividen en diversas líneas prioritarias de trabajo: 
- proyectos o programas de fortalecimiento del tejido productivo: la mayor parte de los proyectos que se desarrollan en este campo están orientados a fortalecer el tejido productivo en alguna de estas modalidades: incubadoras de empresas, apoyo tecnológico sectorial, diversificación productiva; apoyo a micro-emprendimientos; y optimización de la calidad de los proceso.

- proyectos o programas de desarrollo social y comunitario: Los temas vinculados al desarrollo social y comunitario más frecuentes son: vivienda económica, medio ambiente, educación comunitaria e infraestructura urbana.

\section{Metodología}

La investigación adoptó una estrategia de estudio de caso $^{3}$, basada en fuentes primarias y desde la perspectiva de la universidad. En cuanto a su naturaleza y características, las fuentes utilizadas pueden ser clasificadas en dos tipos de documentos: los políticonormativos y los informes cuantitativos. Entre los primeros, los político-normativos, se analizó el Plan Estratégico Institucional (2002), los denominados Programas Institucionales regionales de la UNCPBA (2003), las Resoluciones y Ordenanzas provenientes de la Secretaría de Ciencia, Arte y Tecnología (2001-2008 que fijan los objetivos de la investigación y vinculación en la institución, los Programas Centrales del Rectorado (Polos Tecnológicos, etc.) y los Acuerdos y/o Convenios celebrados con Municipios, Empresas, Organizaciones de la Sociedad Civil e instituciones educativas de otros niveles del ámbito local (2001-2008). Los otros documentos, son informes provenientes de las dependencias centrales del Rectorado (especialmente de la Secretaría de Ciencia, Arte y Tecnología) y los efectuados por funcionarios del rectorado ante el Consejo Superior, que aportan,

\footnotetext{
${ }^{3}$ Esta metodología, como modalidad de investigación, persigue el entendimiento cabal y la comprensión en profundidad de un fenómeno en escenarios individuales para descubrir relaciones y conceptos importantes y así poder obtener una percepción más completa del objeto de estudio, considerándolo como una entidad holística. En ese sentido, el estudio de caso permite un examen detallado, completo e intensivo de una situación/ sujeto/evento (COLÁS, 1994) en su propio contexto y desde una perspectiva integral (GAO, 1990).
}

recopilaciones estandarizadas de tipo anual que expresan en forma cuantitativa (a través de estadísticas e indicadores) los posibles aportes de la UNCPBA al desarrollo local, por ejemplo, por los Centros de Investigación y/o los avances registrados en los convenios /acuerdos firmados con instituciones/empresas del ámbito local.

\section{Transformaciones en las universidades argentinas}

Antes de comenzar con el estudio de caso resulta necesario realizar una breve descripción del contexto en el cuál las universidades fueron implementando sus estrategias de vinculación universitaria. En principio, se puede señalar que las relaciones universidadsociedad se fueron resignificando en función de los procesos económicos y políticos nacionales. La década del ' 90 caracterizada por políticas de corte neoliberal impactaron en todas las áreas económicas, políticas y sociales de la Argentina. De esta forma, la educación superior no escapó a estas tendencias y fue protagonista de profundas transformaciones que tuvieron como correlato la disminución del presupuesto público orientado a la educación superior.

Este escenario, produjo una fractura en la relación del Estado y las universidades, y entre éstas y la sociedad. Si bien, la responsabilidad de las universidades con la sociedad estuvo presente desde los orígenes mismos de las universidades latinoamericanas, el nuevo contexto de restricción financiera desafió a las universidades a buscar recursos externos para solventar sus actividades de docencia e investigación. Es así, que la función de extensión universitaria cedió espacio hacia actividades que estaban más relacionadas la obtención de recursos, en donde los actores privados pasaron a ser los actores deseables de la relación universidad-sociedad. Aquella imagen de universidad comprometida con las cuestiones sociales cedió espacio a una universidad con características más de tipo empresarial.

Sin embargo, el período que se inicia en la última década, instaló nuevamente la discusión del rol de las universidades en la transformación social y económica de la sociedad. La fuerte crisis atravesada en la 
Argentina implicó que todas las instituciones públicas, entre ellas, las instituciones educativas volvieran a replantear su responsabilidad ante la sociedad. En este marco contextual, entre los años 2003 y 2004 las universidades de gestión pública argentinas comenzaron a implementar diversas estrategias que tuvieran un mayor impacto en sus respectivos territorios. De las estrategias centradas en la búsqueda de recursos per se se pasó a estrategias que buscaban responder a distintas demandas de la sociedad.

\subsection{Estrategias de las universidades argentinas}

Actualmente, en las universidades argentinas de gestión pública coexisten estrategias de promoción de la oferta tecnológica con estrategias universitarias más de tipo horizontal que tienen como objetivo articular diversos actores para la resolución de problemáticas del ámbito regional. Estas últimas estrategias se establecen, por un lado, través de la modalidad de proyecto y en otros casos a través de programas institucionales más amplios. En algunos casos, bajo la órbita de las Secretarías de Extensión y en otros bajo las Secretarías de Ciencia y Tecnología. Por ejemplo, la Universidad Nacional del Sur (UNS) ${ }^{4}$ a partir del año 2004 implementó los denominados Proyectos de Grupo de Investigación de Interés Regional (PGI-TIR) ${ }^{5}$ con el objetivo de desarrollar proyectos de investigación orientados a producir conocimiento con un alto impacto en el sector social y productivo de la región. Dichos proyectos tienen una duración de dos años y uno de los requisitos es que al menos participe una institución asociada y el objetivo es la generación de conocimiento científico o tecnológico con alto impacto directo en el sector social y/o productivo regional. La inclusión de una institución como participante del proyecto contribuye a la consolidación de los vínculos con actores del ámbito regional y la creación de conocimiento con la comunidad. Esta modalidad aún hoy sigue vigente.

\footnotetext{
${ }^{4}$ La UNS creada en 1954 es una universidad de tamaño mediano del interior de la Provincia de Buenos Aires.

${ }^{5}$ Las líneas de trabajo definidas como prioritarias fueron: Infraestructura y Servicios Públicos, Producción de Bienes y Servicios, Problemática Social, Cultural y Educativa, Medioambiente y Calidad de Vida, Desarrollo Local y Administración Municipal.
}

Por su parte, la UNCPBA ${ }^{6}$ implementó una estrategia para contribuir al desarrollo bajo la modalidad de programa. Esta experiencia resulta interesante dado que los denominados Programas Institucionales (compuesto por cuatro programas temáticos) constituyen una política específica definida por la universidad para contribuir a los procesos de desarrollo local. Como veremos a continuación, dicha estrategia permitió dar respuesta a distintas demandas de la sociedad.

\section{La UNCPBA y los Programas Institucio- nales}

La UNCPBA, creada en 1974 a través de la Ley 20.753 sancionada por el Congreso Nacional, tiene tres sedes regionales: la sede central, asiento del Rectorado, en la ciudad de Tandil y las sedes de Azul y Olavarría, así como una subsede en Quequén, Partido de Necochea. Alrededor de 13.500 alumnos se distribuyen en 11 unidades académicas, en las que se dictan 16 carreras cortas, 46 carreras de grado, 11 carreras de articulación; 23 carreras de postgrado y 3 diplomaturas. Un rasgo que identifica y destaca a la UNCPBA es la fortaleza de su sistema científico-tecnológico. De acuerdo a la última información oficial disponible proveniente del relevamiento efectuado por la Secretaría de Ciencia, Arte y Tecnología del rectorado, se indica que la universidad posee una dotación de 709 investigadores y 218 becarios de distintos organismos nacionales y provinciales, particularmente CONICET y Comisión de Investigaciones Científicas de la Provincia de Buenos Aires (CIC), dato ilustrativo y relevante si se considera que la planta docente se estima en unas 1000 personas físicas. Ahora bien, el área de influencia de la UNCPBA, de acuerdo a la mencionada Ley de creación $N^{\circ}$ 20.753, está constituida por 19 municipios localizados en el centro de la provincia, entre los que se distinguen tres categorías de ciudades: "pueblos grandes" (de 2.000 a 20.000 habitantes), "ciudades pequeñas"

\footnotetext{
${ }^{6}$ Para un análisis comparado ver Di Meglio y Harispe (2013) "Instrumentos de vinculación universitaria para el desarrollo regional." El caso de las universidades argentinas de gestión pública de tamaño mediano. In: XI Congreso Nacional de Ciencia Política, organizado por la Sociedad Argentina de Análisis Político y la Universidad Nacional de Entre Ríos.
} 
(20.000 a 50.000 habitantes) y "aglomeraciones de tamaño intermedio" (ATI). Entre estas últimas se ubican Tandil, Olavarría, Azul y Necochea donde se emplazan las sedes de la universidad. Por tanto, el estudio analizará en forma genérica las estrategias de "vinculación universitaria" en función de este ámbito de influencia regional.

\subsection{Programas Institucionales de la UNCPBA}

El cambio más significativo orientado a restablecer, y aún profundizar, el rol clave que tuviera la UNCPBA en la región en su período fundacional fue la creación en el año 2003 de los "Programas Institucionales" entendidos como una estrategia fundamental para el desarrollo local-regional, al considerar que permitirían movilizar la capacidad de investigación de la Universidad, mediante acciones convergentes orientadas al abordaje rápido y eficaz de problemas de trascendencia para la actividad económica y la calidad de vida de la comunidad regional. Los Programas se elaboraron pensando en la necesidad de promover la articulación y los vínculos de la Universidad con actores e instituciones del sector público y privado en los diferentes niveles. Fueron cuatro los Programas Institucionales creados: Alimentos, Ordenamiento Ambiental y Patrimonio Natural y Cultural, Apoyo a la Gestión Pública y Apoyo a la Actividad Productiva, de los cuales, los últimos dos fueron los que mayores resultados produjeron en términos cuantitativos y cualitativos.

\subsubsection{Programa Institucional de Apoyo a la Gestión Pública}

En relación al Programa de Apoyo a la Gestión Pública su objetivo fue el de constituirse en un canal de comunicación entre la Universidad y el sector público definido como todo lo concerniente a los intereses y la satisfacción de las necesidades de la comunidad, lo que involucra a un conjunto de actores que trascienden la esfera gubernamental e incorporan también a las Organizaciones Públicas no Estatales de nivel local, regional, provincial o nacional.

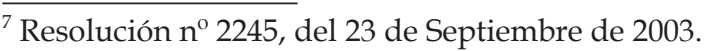

Entre los principios del Plan de Acción 2004 se remarcó fuertemente la necesidad de generar lazos que permitieran impulsar el desarrollo local. Según dicho documento "Los principios filosóficos del Programa Institucional Apoyo a la Gestión Pública tienen estrecha relación con los lineamientos establecidos en el documento titulado "Pour une citoyenneté responsable de l'enseignement supérieur", (París, Junio 2003), en el marco de la convención organizada por la Organización de Naciones Unidas para la Educación, la Ciencia y la Cultura, con representantes de universidades de todo el mundo. Allí se señala que en el actual proceso de globalización, caracterizado por la preeminencia de lo económico y financiero, la Universidad debe cumplir un nuevo rol, pues el tradicional quedó superado por los cambios en curso.

Así, la Universidad debe sustituir su cultura tradicional, por una nueva basada en la pertinencia social de su proyecto, y debe dirigirse a construir un humanismo universalista y una ciudadanía responsable, plural y solidaria. Ello significa integrar todos los componentes sociales y todas las dimensiones (científica, tecnológica, económica, social, ecológica, ética, política, educativa y cultural), y a partir de una de sus misiones centrales, la producción del conocimiento, lograr que este sea útil a la sociedad" (Ord. UNCPBA 3018/03).

Según lo establecido en el documento arriba citado, el objetivo específico del Programa fue promover la articulación de la Universidad con actores e instituciones del sector público, tanto a nivel local, como regional, provincial y nacional, a través de diversas actividades como proyectos de investigación y desarrollo, capacitación y formación de recursos humanos, servicios y asesorías, entre otras posibilidades. El Programa se constituía, de esa manera, en un canal de comunicación con el sector público, tanto a nivel gubernamental como de las Organizaciones Públicas no Estatales, que requirieran de la Universidad su apoyo y el de sus recursos humanos y técnicos, a través de la elaboración de iniciativas y/o proyectos - de los conocimientos científicos-tecnológicos disponibles en la Universidad, en función de su potencial de aplicación a diversas problemáticas sociales.

Los objetivos estratégicos del Programa fueron: 
- El fortalecimiento del desarrollo socioeconómico y educativo-cultural de la región

- El mejoramiento de la calidad de vida de la población

- La cooperación con el sector público, gubernamental y no gubernamental, a fin de contribuir en su mejoramiento y eficiencia

- El fortalecimiento de los lazos de comunicación y trabajo conjunto con dichos sectores, a nivel local y regional, provincial y nacional.

Para lograr dichos objetivos, se debía apuntar a:

- Promover el co-financiamiento de proyectos deinvestigación y desarrollo, y otras acciones relevantes y estratégicas, sistemáticas y continuas, con instituciones públicas de diversa naturaleza, o con empresas o fundaciones que tengan como objetivos los antes mencionados.

- Formalizar dichas actividades en el marco de convenios específicos firmados a tal fin.

- Profundizar los cambios culturalesinstitucionales para fortalecer la capacidad de la universidad para dar respuesta a las demandas provenientes de esos sectores.

- Potenciar la creciente articulación entre los actores e instituciones que conforman el sector público.

Redefiniendo el concepto de lo público se resolvió dividir el programa en dos subprogramas; por un lado: el subprograma apoyo a las organizaciones públicas estatales y apoyo a las organizaciones públicas no estatales. Englobando en el primero de ellos a las estructuras administrativas del estado en sus diferentes esferas (nacional, provincial, municipal) y por otro, en una ampliación del criterio aceptado como espacio público, a las asociaciones civiles que de origen privado contribuyen en la esfera de lo público.

Por otra parte, el programa incluyó aspectos específicos emanados del régimen político-administrativo municipal, provincial y nacional, en cuanto a asesoramiento técnico y desarrollos conjuntos vinculados a diferentes problemáticas que permitan:

- el mejoramiento y modernización de sus sistemas de administración y gestión,

- una ampliación de su capacidad de intervención activa en beneficio de todos los sectores de la población,

- la calidad y eficiencia en los procesos de gestión,

- promover la articulación y vinculación entre instituciones que participan en el proceso de generación, difusión y absorción de conocimientos e innovaciones;

- contribuir al desarrollo de programas para la capacitación de Recursos Humanos y la modernización tecnológica;

- colaborar en la mejora o implementación de diseños institucionales que atiendan a las especificidades de cada organismo.

\subsubsection{Proyectos: tipo y temas}

En ese sentido, en la Convocatoria del año 2004 se presentaron un total de 30 (treinta) iniciativas y como resultado, el Comité Ejecutivo del Programa elevó a la Comisión de Representantes, cuya tarea es evaluar el desempeño y la implementación del programa, su propuesta de aprobación de un total de 19 (diecinueve) proyectos. La misma contenía la aprobación y financiamiento de 12 (doce) proyectos, y el reconocimiento y aval institucional del Programa de otros 7 (siete) proyectos. La propuesta fue aprobada por unanimidad.

Así, en el marco del Programa Institucional de Apoyo a la Gestión Pública se privilegió, por un lado, el financiamiento de proyectos cuyo objetivo era un alto impacto social inmediato considerando la situación de vulnerabilidad en que se encontraban ciertos sectores de la sociedad. En ese contexto, se financiaron proyectos tales como: 
Tabla 1 - Proyectos aprobados en la Convocatoria Año 2004 según tipo de proyecto y actores internos y externos participantes.

\begin{tabular}{|c|c|c|c|}
\hline \multirow{2}{*}{ Proyecto } & \multirow{2}{*}{ Tema } & \multicolumn{2}{|l|}{ Participantes } \\
\hline & & Actores internos y externos & Descripción \\
\hline $\begin{array}{l}\text { "Proyecto social } \\
\text { y educativo } \\
\text { Barriadas" }\end{array}$ & $\begin{array}{l}\text { Educación } \\
\text { comunitaria } \\
\text { e inclusión } \\
\text { social }\end{array}$ & $\begin{array}{l}\text { Secretaría de Bienestar y } \\
\text { Extensión Universitaria y } \\
\text { bibliotecas públicas ("Paula } \\
\text { Albarracín", "Martín } \\
\text { Fierro", "Bepo Ghezzi, entre } \\
\text { otras), ONG's (Asociación } \\
\text { Casa del Niño en la Calle, } \\
\text { APONOVID) y el Centro de } \\
\text { Oficios Universidad Barrial. }\end{array}$ & $\begin{array}{l}\text { Se constituyó una red de } \\
\text { instituciones - educativas, } \\
\text { sociales, deportivas - que a } \\
\text { través de la actividad conjunta } \\
\text { abordaron en las zonas } \\
\text { periféricas de la ciudad el } \\
\text { problema de la exclusión social } \\
\text { y educativa a través de un } \\
\text { proyecto que abarcaba múltiples } \\
\text { dimensiones: pedagógicas, } \\
\text { culturales, deportivas. }\end{array}$ \\
\hline $\begin{array}{l}\text { "Talleres Socio- } \\
\text { Educativos en los } \\
\text { Barrios" }\end{array}$ & $\begin{array}{l}\text { Educación } \\
\text { comunitaria } \\
\text { e inclusión } \\
\text { social }\end{array}$ & $\begin{array}{l}\text { Facultad de Ciencias } \\
\text { Humanas y la Asociación } \\
\text { Civil Solidaria Na Amanda } \\
\left(\text { ONG n }{ }^{\circ} 3989\right) .\end{array}$ & $\begin{array}{l}\text { Proyectos educativos - sociales } \\
\text { orientados a la inclusión social } \\
\text { de familias en situación de } \\
\text { riesgo (Barriadas se dirigía a la } \\
\text { niñez y adolescencia) a través } \\
\text { del desarrollo de actividades } \\
\text { artísticas, literarias, de educación } \\
\text { física recreativas y apoyo } \\
\text { psicológico gratuito a través de } \\
\text { profesionales de la facultad. }\end{array}$ \\
\hline $\begin{array}{l}\text { "Actividad social } \\
\text { veterinaria en los } \\
\text { barrios de Tandil" }\end{array}$ & $\begin{array}{l}\text { Medio } \\
\text { ambiente }\end{array}$ & $\begin{array}{l}\text { Facultades de Ciencias } \\
\text { Veterinarias, Humanas y } \\
\text { Arte, a las Secretarías del } \\
\text { Rectorado, la Municipalidad } \\
\text { de Tandil y al Departamento } \\
\text { de Zoonosis del Ministerio } \\
\text { de Salud de la Provincia de } \\
\text { Buenos Aires. }\end{array}$ & $\begin{array}{l}\text { Actividades vinculadas a las } \\
\text { temáticas de la salud pública y } \\
\text { el cuidado del medio ambiente } \\
\text { en comunidades barriales (riesgo } \\
\text { alimentario, de la prevención de } \\
\text { la zoonosis y de la contaminación } \\
\text { del agua para consumo humano). }\end{array}$ \\
\hline $\begin{array}{l}\text { "Capacitación en } \\
\text { Informática para } \\
\text { Jóvenes" }\end{array}$ & $\begin{array}{l}\text { Educación } \\
\text { comunitaria } \\
\text { e inclusión } \\
\text { social }\end{array}$ & $\begin{array}{l}\text { Facultad de Ciencias Exactas } \\
\text { y Escuelas Primarias, } \\
\text { secundarias y especiales. }\end{array}$ & $\begin{array}{l}\text { Dividido en dos subprogramas: } \\
\text { uno orientado para Jóvenes con } \\
\text { necesidades educativas especiales, } \\
\text { el otro dirigido a Jóvenes en } \\
\text { situación de vulnerabilidad } \\
\text { social. cursos de Informática } \\
\text { para alumnos de esas escuelas } \\
\text { que carecían de medios y } \\
\text { recursos humanos para acceder } \\
\text { a las modernas tecnologías de la } \\
\text { Información y la Comunicación, } \\
\text { atendiendo a una población en } \\
\text { situación de vulnerabilidad social. }\end{array}$ \\
\hline $\begin{array}{l}\text { "Base de Datos } \\
\text { Empresaria y } \\
\text { Sustitución de } \\
\text { Importaciones" }\end{array}$ & $\begin{array}{l}\text { Sistema } \\
\text { económico- } \\
\text { productivo }\end{array}$ & $\begin{array}{l}\text { Facultad de Ingeniería } \\
\text { con la participación y } \\
\text { cofinanciamiento de la } \\
\text { Municipalidad de Olavarría, } \\
\text { la Cámara Empresaria de } \\
\text { dicha localidad y la filial de } \\
\text { la Sociedad Rural. }\end{array}$ & $\begin{array}{l}\text { Generar una fuente de } \\
\text { información que permitiera } \\
\text { conocer el sector productivo y } \\
\text { de servicios de la localidad de } \\
\text { Olavarría, }\end{array}$ \\
\hline $\begin{array}{l}\text { "Relevamiento } \\
\text { normativo en la } \\
\text { ciudad de Azul" }\end{array}$ & $\begin{array}{l}\text { Sistema } \\
\text { jurídico- } \\
\text { normativo }\end{array}$ & $\begin{array}{l}\text { Facultad de Derecho y la } \\
\text { Municipalidad de Azul. }\end{array}$ & $\begin{array}{l}\text { Recopilación jurídica a nivel } \\
\text { local, su sistematización y del } \\
\text { libre acceso para la ciudadanía. }\end{array}$ \\
\hline
\end{tabular}

Fuente: Elaboración propia en base a PIAGP, 2007. 
A partir del año 2007 se incluyeron en el nuevo Plan de Acción del Programa Institucional otros proyectos en acuerdo con diferentes municipios de la región, tales como "Recopilación e Integración de la Información Técnica Disponible sobre las actividades agro-industriales y portuarias en la zona del Estuario del Río Quequén", desarrollado por investigadores del Instituto de Física Arroyo Seco, de la Facultad de Ciencias Exactas en conjunto con la Municipalidad de Necochea, o el titulado "Articulación institucional. Consolidación del destino turístico rural de Los Pinos y San Agustín" radicado en la Facultad de Ciencias Humanas y desarrollado en forma conjunta con la Municipalidad de Balcarce.

\subsubsection{Resultados parciales}

Acerca de los resultados parciales de este programa, la Coordinación del mismo con fecha 11 de Mayo de 2007 elevó al Consejo Superior de la UNCPBA un detallado análisis de lo actuado, registrando el informe los siguientes datos. Los recursos externos generados por los proyectos desarrollados en el Plan de Acción 2005/2006 provinieron de los siguientes organismos (PIAGP, 2007, p. 5-7):

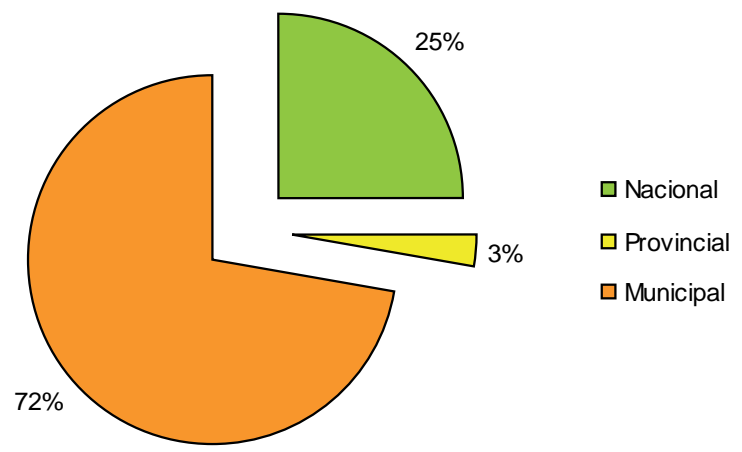

Gráfico 1 - Recursos externos percibidos según organismo, 2005-2006

Fuente: elaboración propia en base a PIAGP, 2007.

Sobre un total de $\$ 165.207,50$, el Estado Nacional aportó \$41.200,00, el ámbito provincial \$ 4.750,00 y \$ 119.257,50 el Estado Municipal. Este monto total significa que se ha triplicado la relación entre presupuesto asignado por el PIAGP y los montos externos generados. Además, la mayor participación corresponde al Estado municipal.

En cuanto a los niveles de participación en proyectos 2005/2006 se señala a continua- ción, en términos cuantitativos, la cantidad de personal e instituciones participantes en los proyectos desarrollados en el Plan de Acción 2005-2006.

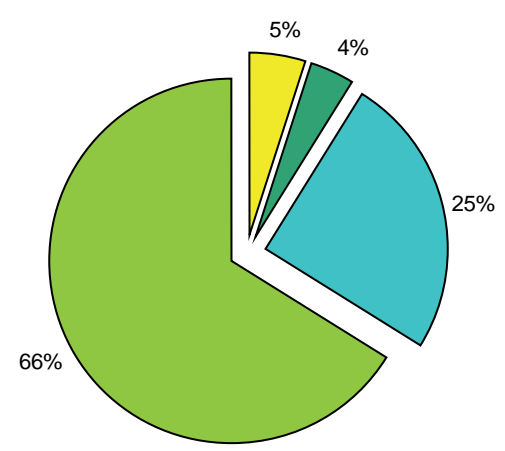

$\square$ Graduados

$\square$ No docentes

$\square$ Docentes

$\square$ Estudiantes

Gráfico 2 - Personal participante en el PIAGP-2005-2006

Fuente: elaboración propia en base a PIAGP, 2007.

El total de personal participantes asciende a 187, de los cuáles 9 son graduados, 7 no docentes, 7 asesores externos, 45 son docentes y 119 estudiantes (PIAGP, 2007, p. 6).

En cuanto a los organismos participantes, el total es de 49: 1 organismo público nacional, 5 provincial y 15 municipal, 16 escuelas, 5 bibliotecas, 5 ONG y 2 empresas privadas (PIAGP, 2007, p. 6-7). La mayor participación estuvo representada por municipios y escuelas de distintos niveles educativos, hecho que está vinculado al tipo de proyectos que se privilegió en las convocatorias analizadas. Los tópicos educación comunitaria e inclusión social y sistematización de información territorial fueron las problemáticas más abordadas por los proyectos. De los proyectos de educación comunitaria participaron organismos externos como escuelas, bibliotecas, ONGs y de los proyectos más orientados al ámbito económico-productivo se destaca la participación de municipios y empresas. 


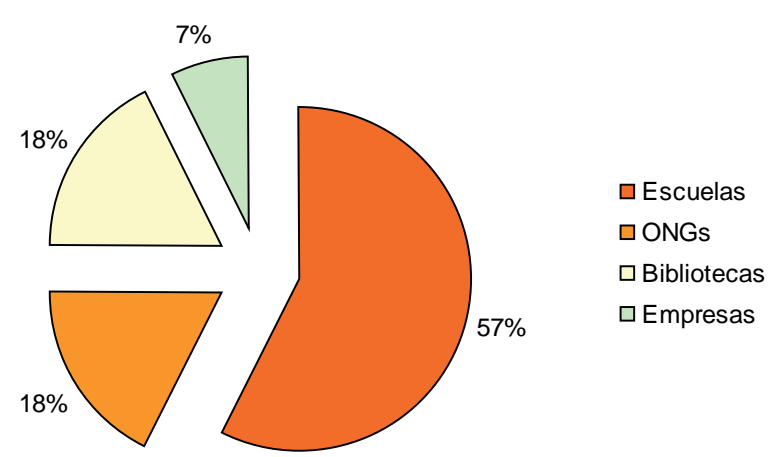

Gráfico 3 - Participación de organismos externos por tipo de destinatario

Fuente: elaboración propia en base a PIAGP, 2007.

Estos datos concretos dejan en evidencia los alcances de este programa institucional conjuntamente con la diversidad de líneas abordadas y los actores territoriales y extraterritoriales implicados, así como los claustros involucrados.

6.2 Programa Institucional de Apoyo a la Actividad Productiva

Con la mira puesta en lograr un doble objetivo por un lado, dar respuesta a las demandas percibidas por la sociedad, y por otro con el objetivo de asumir una actitud propositiva es que se crea el Programa de Apoyo a la Actividad Productiva (PIAAP) a través del cual la Universidad se propone contribuir a la generación regional de riqueza por la producción y comercialización de bienes y servicios, coordinando y potenciando actividades científicas y tecnológicas orientadas a responder a demandas provenientes de empresas y organismos, y también a explorar nuevas áreas, metodologías, o técnicas de producción y de gestión, a estimular la generación de conglomerados productivos o clúster, y a implementar modalidades que ayuden al nacimiento de nuevas empresas en áreas de actividad prometedoras.

Este programa fue creado por la Ordenanza $n^{\circ} 2301$ del Consejo Superior de la Universidad el día 5 de Diciembre de 2003. En su Plan de Acción el PIAAP procuraba atender tanto a demandas formuladas por empresas como también a identificar oportunidades, donde la palabra oportunidad se refiere tanto a la posibilidad de crecimiento de una actividad subdimensionada respecto a su potencialidad o a la posibilidad de introducir nuevas modalidades técnicas $u$ organizacionales capaces de generar un fuerte crecimiento en actividades ya afianzadas. Los proyectos, acciones y actividades de formación en el marco del PIAAP se concretaron alrededor de cuatro ejes centrales: a) Parque Científico Tecnológico, b) Regionalización Productiva, c) Mejora de la Competitividad Productiva y d) Nuevos emprendimientos de base tecnológica.

\subsubsection{Proyectos ejecutados: tipo y temas}

En el Expediente $n^{\circ}, 1-33819 / 2009$ anexo de la Ordenanza $n^{\circ} 3376$ del Consejo Superior, se aprueba el informe de lo actuado hasta esa fecha por el Programa Institucional de Apoyo a la Actividad Productiva:

\section{A - Proyectos Aprobados en la Primera Convocatoria Interna 2005.}

Un total de ocho (8) proyectos fueron aprobados por el Comité Evaluador del Programa Institucional de Apoyo a la Actividad Productiva (PIIAP) en la Primera Convocatoria Anual para el financiamiento de Proyectos y Acciones Específicas, que comenzaron a desarrollarse a principios del año 2005: 
Tabla 2 - Proyectos aprobados en la Convocatoria Año 2005 según tipo de proyecto y actores internos y externos participantes.

\begin{tabular}{|c|c|c|c|}
\hline \multirow[b]{2}{*}{ Proyecto } & \multirow[b]{2}{*}{ Tema } & \multicolumn{2}{|c|}{ Participantes } \\
\hline & & $\begin{array}{l}\text { Actores internos y } \\
\text { externos }\end{array}$ & Descripción \\
\hline $\begin{array}{l}\text { "Programa de Asistencia } \\
\text { Técnica Cooperativa } \\
\text { Impopar" }\end{array}$ & $\begin{array}{l}\text { Competitividad } \\
\text { Productiva }\end{array}$ & $\begin{array}{l}\text { Facultad de Ingeniería } \\
\text { y la Secretaría General }\end{array}$ & $\begin{array}{l}\text { Elaboración de documentación } \\
\text { técnica que respaldara los } \\
\text { equipos fabricados en la fábrica } \\
\text { recuperada y actual cooperativa } \\
\text { Impopar. }\end{array}$ \\
\hline "Publicación Inteligente" & $\begin{array}{l}\text { Compettitivad } \\
\text { productiva }\end{array}$ & \begin{tabular}{|l|} 
Facultad de Ciencias \\
Exactas y la Facultad de \\
Ciencias Económicas \\
\end{tabular} & $\begin{array}{l}\text { Se desarrolló una "Publicación } \\
\text { Inteligente" como "Start Up" de } \\
\text { origen universitario }\end{array}$ \\
\hline $\begin{array}{l}\text { "Desarrollo de un } \\
\text { simulador de empresas } \\
\text { ganaderas de base } \\
\text { pastoril" }\end{array}$ & $\begin{array}{l}\text { Competitividad } \\
\text { productiva }\end{array}$ & $\begin{array}{l}\text { Facultades de } \\
\text { Ciencias Veterinarias, } \\
\text { Ciencias Exactas y } \\
\text { el Área de Dinámica } \\
\text { Organizacional } \\
\end{array}$ & $\begin{array}{l}\text { Se desarrolló un modelo } \\
\text { simulador que función con } \\
\text { todos los datos de un modelo } \\
\text { de invernada y ha sido testeado } \\
\text { por el grupo de desarrolladores. }\end{array}$ \\
\hline $\begin{array}{l}\text { "Desarrollo de un } \\
\text { prototipo de software } \\
\text { para la captura y } \\
\text { centralización de datos } \\
\text { sobre producción de } \\
\text { bovinos de carne." }\end{array}$ & $\begin{array}{l}\text { Competitividad } \\
\text { productiva }\end{array}$ & $\begin{array}{l}\text { Facultad de } \\
\text { Veterinarias }\end{array}$ & $\begin{array}{l}\text { Se desarrolló un prototipo } \\
\text { para cristalizar la idea de un } \\
\text { software que provea soluciones } \\
\text { informáticas a veterinarios y } \\
\text { productores agropecuarios } \\
\text { vinculados con la producción } \\
\text { de carne bovina, y que permita } \\
\text { aumentar la productividad y } \\
\text { calidad de gestión. }\end{array}$ \\
\hline $\begin{array}{l}\text { "Realización de un } \\
\text { estudio de factibilidad y } \\
\text { plan de negocios para la } \\
\text { instalación de una Planta } \\
\text { Piloto de procesamiento } \\
\text { de suero lácteo" }\end{array}$ & $\begin{array}{l}\text { Competitividad } \\
\text { Productiva }\end{array}$ & $\begin{array}{l}\text { Facultad de Ciencias } \\
\text { Veterinarias y } \\
\text { la Fundación } \\
\text { Universidad Empresa } \\
(\text { FUNIVEMP) }\end{array}$ & $\begin{array}{l}\text { Se avanzó en la construcción de } \\
\text { una Planta Semi-Industrial (PSI) } \\
\text { a partir de recursos aportados } \\
\text { por inversores privados y otros } \\
\text { obtenidos por la empresa en el } \\
\text { sistema crediticio. }\end{array}$ \\
\hline $\begin{array}{l}\text { "Acciones tendientes a } \\
\text { la difusión del PCT de la } \\
\text { UNCPBA" }\end{array}$ & $\begin{array}{l}\text { Regionalización } \\
\text { productiva }\end{array}$ & $\begin{array}{l}\text { Facultades de Ciencias } \\
\text { Económicas y Exactas } \\
\text { y el Área de Dinámica } \\
\text { Organizacional }\end{array}$ & $\begin{array}{l}\text { Se buscó posicionar el PCT a } \\
\text { nivel nacional e internacional. }\end{array}$ \\
\hline $\begin{array}{l}\text { "Conformación del Polo } \\
\text { Agroindustrial: Acciones } \\
\text { tendientes a extender } \\
\text { el PCT de la UNCPBA } \\
\text { hacia el sector de la } \\
\text { Producción y Sanidad } \\
\text { Agropecuaria" }\end{array}$ & $\begin{array}{l}\text { Regionalización } \\
\text { productiva }\end{array}$ & \begin{tabular}{|l|} 
Facultad de Ciencias \\
Veterinarias y \\
Área de Dinámica \\
Organizacional con \\
empresas asociadas al \\
Polo Agropecuario- \\
Industrial $^{9}$ \\
\end{tabular} & $\begin{array}{l}\text { Se fomentaron instancias de } \\
\text { interacción entre los sectores } \\
\text { científico, académico y } \\
\text { empresarial, tendientes al } \\
\text { desarrollo productivo local } \\
\text { y regional en respuesta a las } \\
\text { demandas de la sociedad. } \\
\end{array}$ \\
\hline $\begin{array}{l}\text { "Gestión Estratégica de } \\
\text { Recursos Humanos en } \\
\text { Áreas de Alta Tecnología: } \\
\text { Implicancias de un nuevo } \\
\text { contrato psicológico en } \\
\text { el desarrollo de clúster } \\
\text { de Software y Servicios } \\
\text { Informáticos" }\end{array}$ & $\begin{array}{l}\text { Regionalización } \\
\text { productiva }\end{array}$ & & $\begin{array}{l}\text { Este proyecto procuró una } \\
\text { caracterización de los recursos } \\
\text { humanos altamente calificados } \\
\text { que típicamente requieren las } \\
\text { empresas deSoftware y Servicios } \\
\text { Informáticos de la Argentina. }\end{array}$ \\
\hline
\end{tabular}

Fuente: elaboración propia en base a PIAPP, 2005.

\footnotetext{
${ }^{8}$ Fundada en el año 1996, la FUNIVEMP - expresión de la Universidad y las empresas del territorios - fue concebida como una herramienta de enlace entre el conocimiento y la producción, se creó con el objetivo de facilitar la articulación entre el sistema científico-tecnológico y la producción, contribuyendo al desarrollo de la investigación y de la extensión, y a la promoción de acciones dirigidas al mejoramiento de la calidad educativa, el mejoramiento de la empresa y la calidad de vida.

${ }^{9}$ En Junio de 2005 se concretó la conformación del Polo Agropecuario-Industrial en conjunto con empresas del ámbito local.
} 


\section{B - Proyectos Aprobados en la Segunda Convocatoria Interna}

Un total de seis (6) nuevos proyectos resultaron aprobados para comenzar a ejecutarse a partir del primer trimestre de 2006. Los proyectos aprobados fueron:

Tabla 3 - Proyectos aprobados en la Convocatoria Año 2006 según tipo de proyecto y actores internos y externos participantes.

\begin{tabular}{|c|c|c|c|}
\hline \multirow[b]{2}{*}{ Proyecto } & \multirow[b]{2}{*}{ Tema } & \multicolumn{2}{|c|}{ Participantes } \\
\hline & & $\begin{array}{l}\text { Actores internos y } \\
\text { externos }\end{array}$ & Descripción \\
\hline $\begin{array}{l}\text { "Programa de mejora } \\
\text { productiva y de calidad } \\
\text { en fábricas de bloques } \\
\text { graníticos de Olavarria" }\end{array}$ & $\begin{array}{l}\text { Competitividad } \\
\text { Productiva }\end{array}$ & $\begin{array}{l}\text { Facultad de Ingeniería, } \\
\text { FUNIVEMP con } \\
\text { agrupación de } \\
\text { fabricantes de bloques } \\
\text { graníticos y la Munici- } \\
\text { palidad de Olavarria. }\end{array}$ & $\begin{array}{l}\text { Se realizaron numerosos } \\
\text { ensayos que permitieron } \\
\text { establecer planes de mejoras en } \\
\text { los procesos productivos y de } \\
\text { gestión. }\end{array}$ \\
\hline $\begin{array}{l}\text { "Promoción de comercio } \\
\text { internacional en } \\
\text { Olavarria" }\end{array}$ & $\begin{array}{l}\text { Compettitivad } \\
\text { productiva }\end{array}$ & $\begin{array}{l}\text { Facultad de Inge- } \\
\text { niería, el Rectorado } \\
\text { de la UNCPBA, la } \\
\text { Secretaría de Desar- } \\
\text { rollo Económico } \\
\text { del Municipio } \\
\text { de Olavarría y } \\
\text { productores locales. }\end{array}$ & $\begin{array}{l}\text { Elaboración de un diagnóstico } \\
\text { que intentó establecer el } \\
\text { potencial exportador de la } \\
\text { región, y en consecuencia } \\
\text { plantear el desarrollo de } \\
\text { distintos cursos de acción en } \\
\text { conjunto con el Municipio. }\end{array}$ \\
\hline $\begin{array}{l}\text { "Desarrollo e implemen- } \\
\text { tación de un programa } \\
\text { de control y recopilación } \\
\text { de información para } \\
\text { establecimientos dedica- } \\
\text { dos a la producción } \\
\text { de carne bovina de la } \\
\text { Región Pampeana" } \\
\end{array}$ & $\begin{array}{l}\text { Competitividad } \\
\text { productiva }\end{array}$ & $\begin{array}{l}\text { Facultades de } \\
\text { Ciencias Económicas } \\
\text { y Ciencias } \\
\text { Veterinarias con } \\
\text { establecimientos } \\
\text { productivos de la } \\
\text { Región. }\end{array}$ & $\begin{array}{l}\text { Se logró desarrollar } \\
\text { e implementar una } \\
\text { estructura y metodología } \\
\text { de trabajo apropiada para el } \\
\text { funcionamiento posterior de la } \\
\text { Unidad de Control de Procesos } \\
\text { Productivos de la Facultad de } \\
\text { Ciencias Veterinarias. }\end{array}$ \\
\hline $\begin{array}{l}\text { "Valorización de } \\
\text { los residuos sólidos } \\
\text { industriales para su } \\
\text { gestión sustentable en la } \\
\text { actividad de fundición de } \\
\text { hierro" }\end{array}$ & $\begin{array}{l}\text { Competitividad } \\
\text { productiva }\end{array}$ & \begin{tabular}{|l|} 
Facultad de Ciencias \\
Humanas a través \\
del Centro de Inves- \\
tigación y Estudios \\
Ambientales (CINEA), \\
FUNIVEMP y la \\
empresa Metalúrgica \\
Tandil S.A. \\
\end{tabular} & $\begin{array}{l}\text { Se efectuó un relevamiento } \\
\text { de los principales insumos y } \\
\text { materias primas de todos los } \\
\text { procesos más significativos. } \\
\text { Posteriormente se planifico } \\
\text { la etapa de muestreo para su } \\
\text { posterior análisis químico. }\end{array}$ \\
\hline $\begin{array}{l}\text { "Gerenciamiento de } \\
\text { material audiovisual de } \\
\text { carácter educativo" }\end{array}$ & $\begin{array}{l}\text { Competitividad } \\
\text { Productiva }\end{array}$ & $\begin{array}{l}\text { Facultad de Arte y } \\
\text { el Área de Dinámica } \\
\text { Organizacional }\end{array}$ & $\begin{array}{l}\text { Las actividades se dirigieron } \\
\text { hacia tres ámbitos diferentes: a) } \\
\text { Confrontación con experiencias } \\
\text { afines; b) Organización } \\
\text { reglamentaria y administración } \\
\text { interna; c) Promoción de la } \\
\text { productora. }\end{array}$ \\
\hline $\begin{array}{l}\text { "Fortalecimiento del } \\
\text { centro de Carreras del } \\
\text { PCT" }\end{array}$ & $\begin{array}{l}\text { Regionalización } \\
\text { productiva }\end{array}$ & \begin{tabular}{|l|} 
Facultad de Ciencias \\
Económicas, el \\
Área de Dinámica \\
Organizacional, \\
FUNIVEMP y \\
empresas asociadas al \\
Polo Informático. \\
\end{tabular} & $\begin{array}{l}\text { Se desarrollan vínculos con } \\
\text { empresas a través de programas } \\
\text { de apoyo y orientación a } \\
\text { estudiantes y graduados. }\end{array}$ \\
\hline
\end{tabular}




\begin{tabular}{|c|c|c|c|}
\hline \multirow[b]{2}{*}{ Proyecto } & \multirow[b]{2}{*}{ Tema } & \multicolumn{2}{|c|}{ Participantes } \\
\hline & & $\begin{array}{l}\text { Actores internos y } \\
\text { externos }\end{array}$ & Descripción \\
\hline $\begin{array}{l}\text { "Desarrollo de las } \\
\text { bases para el diseño } \\
\text { de un Programa de } \\
\text { Emprendedores de base } \\
\text { tecnológica en el marco } \\
\text { del PCT" }\end{array}$ & $\begin{array}{l}\text { Regionalización } \\
\text { productiva }\end{array}$ & $\begin{array}{l}\text { Facultad de Ciencias } \\
\text { Económicas, } \\
\text { FUNIVEMP, el } \\
\text { Área de Dinámica } \\
\text { Organizacional y } \\
\text { empresas incubadas } \\
\text { con procesos de } \\
\text { incubación ad-hoc en } \\
\text { el ámbito del Parque } \\
\text { Científico Tecnológico } \\
(\mathrm{PCT})^{10} \text {. }\end{array}$ & $\begin{array}{l}\text { Se identificaron los factores } \\
\text { subyacentes en el proceso de } \\
\text { formación de emprendedores } \\
\text { y de generación de empresas } \\
\text { innovadoras de base } \\
\text { tecnológica. }\end{array}$ \\
\hline
\end{tabular}

Fuente: elaboración propia en base a PIAPP, 2007.

\subsubsection{Resultados parciales}

Se aprobaron catorce (14) proyectos en total, de los cuáles diez (10) estuvieron orientados a fortalecer la competitividad de los entramados productivos y los otros cuatro (4) se orientaron al fortalecimiento de las áreas institucionales y la creación de nuevos esquemas de relacionamiento horizontal (como la creación del polo agro-industrial bajo la órbita del PCT) en el marco del eje regionalización productiva. Respecto a la participación de organismos externos, se observa una mayor participación de empresas, sobre todo aquellas asociadas al PCT y en menor grado la participación de municipios.

\section{Reflexiones Finales}

En un nuevo contexto en el cuál la universidad rediscute su vínculo con la sociedad y su responsabilidad como institución pública, las universidades argentinas comenzaron a implementar un conjunto de estrategias de vinculación con el objetivo de intervenir en los procesos de desarrollo y responder a ciertas demandas de la sociedad. De las estrategias centradas en la búsqueda de recursos per se o de promoción de la oferta tecnológica se pasó a estrategias que buscan responder a distintas demandas de la sociedad en articulación con actores públicos y privados.

En el modelo analizado se observó el tipo de participación y el aporte de la universidad como un actor con habilidad de movi-

\footnotetext{
${ }^{10}$ En el año 2003 se creó el PCT el cual se conformó inicialmente con empresas productoras de software y servicios informáticos.
}

lizar sus capacidades de investigación en pos del desarrollo local. A diferencia del modelo ofertista la universidad analizada amplió su participación en temas de gobierno (asesorías al Estado), en políticas sociales (educación, salud, organización social) y en innovación productiva (articulación con municipios, cooperativismo, cámaras empresariales) con una mirada más integral del proceso de vinculación universitaria y como un actor del territorio con intereses propios y modalidades específicas de intervención.

A diferencia de la modalidad por proyectos, el tipo de estrategia institucional utilizada funcionó como una política horizontal de intervención y diálogo permanente con múltiples actores locales. En esta misma línea, promovió dos procesos convergentes: un proceso de articulación hacia fuera (en su relación con actores externos) y como una instancia de coordinación hacia adentro (entre las estructuras internas) en la consolidación de proyectos de desarrollo. Asimismo, este proceso derivó en el establecimiento de otro tipo de estrategias de actuación de mayor coordinación como los polos tecnológicos en asociación con actores públicos y privados bajo la órbita del PIAAP.

En este sentido, podemos inferir que las estrategias analizadas constituyen una nueva forma de articulación de la universidad con su entorno asumiendo un papel mucho más activo para la discusión y solución de los problemas de la sociedad en la cual se inserta. Sin embargo, este nuevo rol requirió llevar a cabo procesos de reorganización académicaadministrativa y reformulaciones institucionales y políticas profundas que se abordará en futuras investigaciones. 


\section{Bibliografía}

AROCENA, Rodrigo; SUTZ, Judith. La universidad latinoamericana del futuro. Tendencias, escenarios, alternativas. México: UDUAL, 2001.

BOISIER, Sergio. ¿Hay espacio para el desarrollo local en la globalización? Revista CEPAL, Santiago, n. 86, p. 47-62, 2005

CASAS, Rosalba. El enfoque de redes y flujos de conocimiento en el análisis de las relaciones entre ciencia, tecnología y sociedad. Revista Kairos, San Luis, ano 5, n. 8, 2001.

CIAI, Ariadna et al. La vinculación tecnológica a nivel local. Un desafío para la gestión de ciencia y tecnología. In: Programa de Asistencia en Gestión de Ciencia y Tecnología. Buenos Aires: Secretaría de la Función Pública, 1997.

DAGNINO, Renato; VELHO, Lea. University-Industry Government Relations on the Periphery: The University of Campinas, Brazil. Minerva, London, v. 36, n. 3, p. 229-251, 1998.

DI MEGLIO, Fernanda; HARISPE, Andrés. Instrumentos de vinculación universitaria para el desarrollo regional. El caso de las universidades argentinas de gestión pública de tamaño mediano. In: CONGRESO NACIONAL DE CIENCIA POLÍTICA, 11., organizado por la Sociedad Argentina de Análisis Político y la Universidad Nacional de Entre Ríos, 2013.

FERNÁNDEZ DE LUCIO, Ignacio et al. Las relaciones entre universidad-empresa: entre la transferencia de resultados y el aprendizaje regional. Revista Espacios, Caracas, v. 21, n. 2, 2000.
GIBB, Allen. The enterprise culture and education: understanding enterprise education and its links with small business, entrepreneurship and wider educational goals. International Small Business Journal, v. 11, n. 3, p. 14-34, 1993.

HERNÁNDEZ PÉREZ, Hernando. La Universidad como Actor del Desarrollo Local. Revista Futuros, v. VI, n. 20, 2008.

MOLAS-GALLART, Jordi. Definir, quantificar i finançar la tercera missió: un debat sobre el futur de la Universitat. Revista Coneixement $i$ Societat, Valencia, $\mathrm{n}$. 7, p. 6-27, 2005.

ROFMAN, Adriana; VILLAR, Alejandro. Actores del Desarrollo Local. In: Bloque de Formación de Fundamento de Desarrollo Local, Instituto Nacional de Formación Docente, 2007.

ROFMAN, Adriana. Universidad y Desarrollo Local: Aprendizajes y desafíos. Buenos Aires: Universidad Nacional de General Sarmiento, 2005.

SAGASTIZÁBAL, Leandro. Diseñar una nación. Buenos Aires: Norma, 2002.

STILES, David. Methods in the 1990s: National and Regional Developments and Policy Implications. Public Administration, Oxford, v. 80, n. 4, p. 711-731, 2002.

SUTZ, Judith. Innovación y desarrollo en América Latina, Caracas: Nueva Sociedad, 1997.

VAPÑARSKI, Cesar; GOROJSVSKY, Néstor. El crecimiento urbano en la Argentina. Buenos Aires: Grupo Editor Latinoamericano, 1990.

VELÁZQUEZ, Guillermo. Geografía, calidad de vida y fragmentación en la Argentina de los noventa: análisis regional y departamental. Tandil: UNCPBA, 2001. 
